\title{
On the space of metrics with invertible Dirac operator
}

\author{
Mattias Dahl
}

\begin{abstract}
On a compact spin manifold we study the space of Riemannian metrics for which the Dirac operator is invertible. The first main result is a surgery theorem stating that such a metric can be extended over the trace of a surgery of codimension at least three. We then prove that, if non-empty, the space of metrics with invertible Dirac operators is disconnected in dimensions $n \equiv 0,1,3,7 \bmod 8, n \geq 5$. As corollaries follow results on the existence of metrics with harmonic spinors by Hitchin and Bär. Finally we use computations of the eta invariant by Botvinnik and Gilkey to find metrics with harmonic spinors on simply connected manifolds with a cyclic group action. In particular this applies to spheres of all dimensions $n \geq 5$.
\end{abstract}

Mathematics Subject Classification (2000). 53C27, 57R65, 58J05, 58J50.

Keywords. Eigenvalues of the Dirac operator, surgery.

\section{Introduction}

Let $(M, g)$ be a Riemannian spin manifold, we will always assume that such a manifold comes equipped with an orientation and a spin structure. We denote by $M^{-}$the same manifold with the opposite orientation. The Dirac operator $D^{g}$ is a first order elliptic differential operator acting on smooth sections of the spinor bundle $\Sigma M$. If $M$ has a boundary we will only consider Riemannian metrics on $M$ which have a product structure in a neighbourhood of the boundary.

For a Riemannian manifold $(M, g)$ with boundary $\partial M$ we denote by $\left(M_{\infty}, g\right)$ the same manifold with the half-infinite cylinder $\left([0, \infty) \times \partial M, d x^{2}+\left.g\right|_{\partial M}\right)$ attached along the boundary (here we abuse notation slightly by using the same symbol $g$ for the metric on $M$ and the metric on $M_{\infty}$ ). If $M$ is closed, that is compact with no boundary, we set $\left(M_{\infty}, g\right)=(M, g)$.

We denote by $C_{0}^{\infty}(\Sigma M)$ the space of compactly supported smooth sections of $\Sigma M$. On a complete Riemannian manifold $(M, g)$ we denote by $L^{2}(\Sigma M)$ and $H^{1}(\Sigma M)$ the completions of $C_{0}^{\infty}(\Sigma M)$ with respect to the $L^{2}$-norm $\|\cdot\|$ and the first Sobolev norm. 
If $(M, g)$ is compact without boundary the operator $D^{g}$ has a self-adjoint extension to $L^{2}(\Sigma M)$ with domain $H^{1}(\Sigma M)$. This is a Fredholm operator with discrete spectrum [13, Chapter $3, \S 5]$. If $(M, g)$ is compact with non-empty boundary we consider the Dirac operator $D^{g}$ on the manifold $\left(M_{\infty}, g\right)$ with cylindrical ends. In this case we also have a self-adjoint extension to $L^{2}\left(\Sigma M_{\infty}\right)$ with domain $H^{1}\left(\Sigma M_{\infty}\right)$, see [6, Section 3.6.2].

Now suppose $(M, g)$ is compact, possibly with boundary. The operator $D^{g}$ is invertible with a bounded inverse if and only if it has a spectral gap around 0 , that is if there is an $\varepsilon>0$ such that $\left\|D^{g} \varphi\right\|^{2} \geq \varepsilon\|\varphi\|^{2}$ for all $\varphi \in L^{2}\left(\Sigma M_{\infty}\right)$.

Definition. Let $M$ be a compact spin manifold. We define $R^{\text {inv }}(M)$ to be the set of Riemannian metrics $g$ on $M$ for which $D^{g}$ is invertible with a bounded inverse. By $R^{\mathrm{psc}}(M)$ we denote the set of Riemannian metrics on $M$ with positive scalar curvature.

Let $R(M)$ be set of all Riemannian metrics on $M$. If $M$ is a closed spin manifold, then $R^{\text {inv }}(M)$ is an open subset of $R(M)$ in the $C^{1}$-topology, and if $R^{\text {inv }}(M)$ is non-empty, then it is dense in $R(M)$ in the $C^{k}$-topology for all $k \geq 1$, see [4, Proposition 3.2].

Proposition 1.1. If $g \in R^{\text {inv }}(M)$ then $\left.g\right|_{\partial M} \in R^{\text {inv }}(\partial M)$.

Proof. Suppose that the Dirac operator for $\left.g\right|_{\partial M}$ is not invertible. Then there is a $\varphi \neq 0$ such that $D^{\left.g\right|_{\partial M}} \varphi=0$. If we extend $\varphi$ to the cylindrical end of $\left(M_{\infty}, g\right)$ by parallel transport in the normal direction and then multiply with a cut-off function having small gradient we can construct compactly supported $\psi$ on $M_{\infty}$ for which $\left\|D^{g} \psi\right\|^{2} /\|\psi\|^{2}$ is arbitrarily small.

Definition 1.2. Let $M, N$ be compact spin manifolds without boundary.

(1) Metrics $g^{0}, g^{1} \in R^{\text {inv }}(M)$ are called isotopic if there is a smooth path of metrics $g_{t} \in R^{\text {inv }}(M), t \in \mathbb{R}$, such that $g_{t}=g^{0}$ for $t \leq 0$ and $g_{t}=g^{1}$ for $t \geq 1$.

(2) Metrics $g^{0}, g^{1} \in R^{\text {inv }}(M)$ are called concordant if there is a metric $\bar{g} \in$ $R^{\text {inv }}([0,1] \times M)$ such that $\left.\bar{g}\right|_{\{i\} \times M}=g^{i}, i=0,1$.

(3) Metrics $g^{0} \in R^{\text {inv }}(M), g^{1} \in R^{\text {inv }}(N)$, are called bordant if there is a manifold $W$ and a metric $g^{W} \in R^{\text {inv }}(W)$ so that $\partial\left(W, g^{W}\right)=\left(M, g^{0}\right) \sqcup\left(N^{-}, g^{1}\right)$.

It is immediate that isotopy is an equivalence relation, that concordance and bordance also are equivalence relations will follow from Proposition 2.1.

The Dirac operator is intimately related to the scalar curvature. From the Lichnerowicz formula $\left(D^{g}\right)^{2}=\left(\nabla^{g}\right)^{*} \nabla^{g}+\frac{1}{4} \mathrm{scal}^{g}$ it follows that $R^{\mathrm{psc}}(M) \subset R^{\mathrm{inv}}(M)$. There are corresponding relations psc-isotopic/psc-concordant/psc-bordant for metrics in $R^{\mathrm{psc}}(M)$. The Lichnerowicz formula implies that if two metrics are pscisotopic/psc-concordant/psc-bordant then they are isotopic/concordant/bordant. 
The principal idea of this paper is to study the space $R^{\text {inv }}(M)$ using techniques from the study of $R^{\mathrm{psc}}(M)$. In Section 2 we will look at ways of constructing Riemannian manifolds with invertible Dirac operator, the most powerful of which will be the extension of a metric with invertible Dirac operator to the trace of a surgery of codimension at least 3. The main result of the paper is in Section 3 where we use the Index Theorem to detect non-concordant metrics in $R^{\text {inv }}(M)$ in dimensions $n \equiv 0,1,3,7 \bmod 8, n \geq 5$. The construction of these non-concordant metrics uses known examples of "exotic" metrics in $R^{\mathrm{psc}}\left(S^{n}\right)$ which do not bound metrics in $R^{\mathrm{psc}}\left(D^{n+1}\right)$. This result shows that if $R^{\mathrm{inv}}(M)$ is non-empty, then it is disconnected, which unifies and strengthens results by Hitchin and Bär on the existence of metrics with non-trivial harmonic spinors.

Several questions concerning the relationship between $R^{\text {psc }}(M)$ and $R^{\text {inv }}(M)$ can be formulated. For simply-connected manifolds the solution of the Gromov-Lawson conjecture tells that these spaces are non-empty at the same time, is it further true that the inclusion $R^{\mathrm{psc}}(M) \rightarrow R^{\mathrm{inv}}(M)$ is surjective on $\pi_{0}$ ? Is the inclusion even a homotopy equivalence? To formulate these questions for manifolds with non-trivial fundamental group $\pi$ the space $R^{\text {inv }}(M)$ should be defined as the set of metrics for which all Dirac operators with coefficients in flat $C^{*} \pi$-bundles over $M$ are invertible. Since the Gromov-Lawson-Rosenberg conjecture is known to fail for some groups it seems unclear what to expect of the inclusion $R^{\mathrm{psc}}(M) \rightarrow R^{\mathrm{inv}}(M)$ in general.

In Section 4 we leave the study of $R^{\text {inv }}(M)$. Instead we use computations of the eta invariant by Botvinnik and Gilkey to find metrics with harmonic spinors on simply connected manifolds with a cyclic group action. In particular we find metrics with harmonic spinors on spheres of all dimensions $n \geq 5$.

\section{Constructions}

In this section we will study three constructions of new Riemannian manifolds with invertible Dirac operators from old ones.

2.1. Attaching isometric boundary components. Let $M$ be a manifold with boundary $\partial M$. Suppose that the boundary is a disjoint union $\partial M=\partial^{+} M \sqcup \partial^{-} M \sqcup \partial^{0} M$ where $\partial^{+} M \cong N$ and $\partial^{-} M \cong N^{-}$for some compact spin manifold $N$ and where $\partial^{0} M$ might be empty.

Suppose $g \in R^{\text {inv }}(M)$ is such that $\left.g\right|_{\partial^{+} M}=\left.g\right|_{\partial^{-} M}=h$ for some metric $h$ on $N$. For $t>0$ let $\left(M^{\prime}, g_{t}^{\prime}\right)$ be $(M, g)$ with the cylinder $\left([0, t] \times N, d x^{2}+h\right)$ attached by $\{0\} \times N$ along $\partial^{+} M$ and by $\{t\} \times N$ along $\partial^{-} M$. The manifolds $M^{\prime}$ depend on $t$ but are all diffeomorphic so we identify them. 
Proposition 2.1. Let $\left(M^{\prime}, g_{t}^{\prime}\right)$ be constructed from $(M, g)$ as above. Then there is $T>0$ so that $g_{t}^{\prime} \in R^{\text {inv }}\left(M^{\prime}\right)$ for all $t>T$.

Note that the manifold $M$ is not assumed to be connected.

Proof. Since $g \in R^{\text {inv }}(M)$ there is $\varepsilon^{g}>0$ so that $\left\|D^{g} \varphi\right\|^{2} \geq \varepsilon^{g}\|\varphi\|^{2}$ for all $\varphi \in$ $L^{2}\left(\Sigma M_{\infty}\right)$. Set $\varepsilon=\frac{\varepsilon^{g}}{8}$ and choose $T>0$ so that $t>T$ implies $\frac{6}{t^{2}} \leq \frac{\varepsilon^{g}}{8}$.

Let $t>T$ and take $\varphi \in C_{0}^{\infty}\left(\Sigma M_{\infty}^{\prime}\right)$. Let $\chi:[0, t] \times N \rightarrow[0,1]$ be a smooth function such that $\chi=1$ near $\{0\} \times N, \chi=0$ near $\{t\} \times N$, and $|\operatorname{grad} \chi| \leq \frac{2}{t}$. A straight-forward computation shows that

$$
|D \varphi|^{2} \geq \frac{1}{2}|D(\chi \varphi)|^{2}+\frac{1}{2}|D((1-\chi) \varphi)|^{2}-\frac{3}{2}|\operatorname{grad} \chi|^{2}|\varphi|^{2}
$$

so

$$
\|D \varphi\|_{[0, t] \times N}^{2} \geq \frac{1}{2}\|D(\chi \varphi)\|_{[0, t] \times N}^{2}+\frac{1}{2}\|D((1-\chi) \varphi)\|_{[0, t] \times N}^{2}-\frac{6}{t^{2}}\|\varphi\|_{[0, t] \times N}^{2} .
$$

We define the spinor field $\psi \in C_{0}^{\infty}\left(M_{\infty}\right)$ as follows. On $M$ and on $[0, \infty) \times \partial^{0} M$ we set $\psi=\varphi$. At $\partial^{+} M$ we first attach $\left([0, t] \times N, d x^{2}+h\right)$ along $\{0\} \times N$ and set $\psi=\chi \varphi$ on this piece, followed by $\psi=0$ on the half-infinite cylinder attached along $\{t\} \times N$. In the same way we attach $\left([0, t] \times N, d x^{2}+h\right)$ at $\partial^{-} M$ along $\{t\} \times N$ and there we set $\psi=(1-\chi) \varphi$ followed by $\psi=0$ on the half-infinite cylinder attached along $\{0\} \times N$. Using the above estimate we get

$$
\begin{aligned}
\|D \varphi\|_{M_{\infty}^{\prime}}^{2}= & \|D \varphi\|_{M}^{2}+\|D \varphi\|_{[0, \infty) \times \partial^{0} M}^{2}+\|D \varphi\|_{[0, t] \times N}^{2} \\
\geq & \|D \varphi\|_{M}^{2}+\|D \varphi\|_{[0, \infty) \times \partial^{0} M}^{2} \\
& \quad+\frac{1}{2}\|D(\chi \varphi)\|_{[0, t] \times N}^{2}+\frac{1}{2}\|D((1-\chi) \varphi)\|_{[0, t] \times N}^{2}-\frac{6}{t^{2}}\|\varphi\|_{[0, t] \times N}^{2} \\
\geq & \frac{1}{2}\left(\|D \varphi\|_{M}^{2}+\|D \varphi\|_{[0, \infty) \times \partial^{0} M}^{2}\right. \\
& \left.\quad+\|D(\chi \varphi)\|_{[0, t] \times N}^{2}+\|D((1-\chi) \varphi)\|_{[0, t] \times N}^{2}\right)-\frac{6}{t^{2}}\|\varphi\|_{M_{\infty}^{\prime}}^{2} \\
= & \frac{1}{2}\|D \psi\|_{M_{\infty}}^{2}-\frac{6}{t^{2}}\|\varphi\|_{M_{\infty}^{\prime}}^{2} \\
\geq & \frac{\varepsilon^{g}}{2}\|\psi\|_{M_{\infty}}^{2}-\frac{6}{t^{2}}\|\varphi\|_{M_{\infty}^{\prime}}^{2} \\
= & \frac{\varepsilon^{g}}{2}\left(\|\varphi\|_{M}^{2}+\|\varphi\|_{[0, \infty) \times \partial^{0} M}^{2}+\|\chi \varphi\|_{[0, t] \times N}^{2}+\|(1-\chi) \varphi\|_{[0, t] \times N}^{2}\right) \\
& \quad-\frac{6}{t^{2}}\|\varphi\|_{M_{\infty}^{\prime}}^{2} \\
\geq & \frac{\varepsilon^{g}}{2}\left(\|\varphi\|_{M}^{2}+\|\varphi\|_{[0, \infty) \times \partial^{0} M}^{2}+\frac{1}{2}\|\varphi\|_{[0, t] \times N}^{2}\right)-\frac{6}{t^{2}}\|\varphi\|_{M_{\infty}^{\prime}}^{2} \\
\geq & \left(\frac{\varepsilon^{g}}{4}-\frac{6}{t^{2}}\right)\|\varphi\|_{M_{\infty}^{\prime}}^{2} \\
\geq & \varepsilon\|\varphi\|_{M_{\infty}^{\prime}}^{2} .
\end{aligned}
$$

Since $C_{0}^{\infty}\left(\Sigma M_{\infty}^{\prime}\right)$ is dense in $L^{2}\left(\Sigma M_{\infty}^{\prime}\right)$ this shows that $g_{t}^{\prime} \in R^{\mathrm{inv}}\left(M^{\prime}\right)$. 
Proposition 2.1 has the following corollary.

Corollary 2.2. Concordance and bordance are equivalence relations.

2.2. Generalized cylinders. Let $M$ be a compact spin manifold of dimension $n$ and let $g_{\tau}$ be a smooth curve of metrics on $M$ parametrized by $\tau \in I$, where $I$ is an interval. The product $\bar{M}=I \times M$ equipped with the metric $\bar{g}=d \tau^{2}+g_{\tau}$ is called a generalized cylinder over $M$. We are going to recall some facts about the spinor bundle and the Dirac operator on a generalized cylinder. All these facts are conveniently collected in [5].

The spin structure on $M$ induces in a unique way a spin structure on $\bar{M}$. The spinor bundle on $(\bar{M}, \bar{g})$ is related to the spinor bundle on $\left(M, g_{\tau}\right)$ by $\Sigma_{(\tau, x)} \bar{M}=\Sigma_{x} M$ if $n$ is even and $\Sigma_{(\tau, x)}^{ \pm} \bar{M}=\Sigma_{x} M$ if $n$ is odd. Denote Clifford multiplication on $\Sigma \bar{M}$ by . and Clifford multiplication on $\Sigma M$ by $\bullet_{\tau}$. If $n$ is even we have $X \bullet_{\tau} \varphi=v \cdot X \cdot \varphi$ and if $n$ is odd $X \bullet_{\tau} \varphi= \pm v \cdot X \cdot \varphi$ for $\varphi \in \Sigma^{ \pm} \bar{M}$. Here $v=\partial_{\tau}$ is the normal of $\{\tau\} \times M$ in $(\bar{M}, \bar{g})$.

Let $\varphi$ be a section of $\Sigma \bar{M}$. The Dirac operators on $M$ and $\bar{M}$ are related by

$$
\nu \cdot D^{\bar{g}} \varphi=\left(D^{g_{\tau}}+\frac{n}{2} H-\nabla_{\nu}^{\bar{g}}\right) \varphi .
$$

Here $H$ is the mean curvature of $\{\tau\} \times M$ in $(\bar{M}, \bar{g})$,

$$
H=-\frac{1}{2 n} \operatorname{tr}_{g_{\tau}}\left(\dot{g}_{\tau}\right),
$$

and if $n$ is odd the operator $D^{g_{\tau}}$ acts on sections of $\Sigma \bar{M}$ by $\left(\begin{array}{cc}D^{g_{\tau}} & 0 \\ 0 & -D^{g_{\tau}}\end{array}\right)$. Let $\dot{g}_{\tau}=\partial_{\tau} g_{\tau}$ and define the operator $\mathfrak{D}^{\dot{g}_{\tau}}$ by $\mathfrak{D}^{\dot{g}_{\tau}} \varphi=\sum_{i, j=1}^{n} \dot{g}_{\tau}\left(e_{i}, e_{j}\right) e_{i} \bullet_{\tau} \nabla_{e_{j}}^{g_{\tau}} \varphi$ where $e_{1}, \ldots, e_{n}$ is an orthonormal basis of $T M$. The commutator of $\nabla_{v}^{\bar{g}}$ and $D^{g_{\tau}}$ is given by [5, Equation (23)]

$$
\left[\nabla_{\nu}^{\bar{g}}, D^{g_{\tau}}\right] \varphi=-\frac{1}{2} \mathfrak{D}^{\dot{g}_{\tau}} \varphi+\frac{1}{4} \operatorname{grad}^{g_{\tau}}\left(\operatorname{tr}_{g_{\tau}}\left(\dot{g}_{\tau}\right)\right) \bullet_{\tau} \varphi-\frac{1}{4} \operatorname{div}^{g_{\tau}}\left(\dot{g}_{t}\right) \bullet_{\tau} \varphi .
$$

Now suppose $g_{\tau}, \tau \in[0,1]$, is a smooth curve of metrics in $R^{\text {inv }}(M)$ with $g_{\tau}=g_{0}$ for $\tau$ near 0 and $g_{\tau}=g_{1}$ for $\tau$ near 1 . Define metrics $\bar{g}_{t}$ on $\bar{M}_{t}=[0, t] \times M$ by $\bar{g}_{t}=d \tau^{2}+g_{\tau / t}$ for $t>0$. Since the $\bar{M}_{t}$ are all diffeomorphic we identify them as $\bar{M}$.

Proposition 2.3. Suppose $\left(\bar{M}, \bar{g}_{t}\right)$ is constructed from $M$ and $g_{\tau}$ as above. Then there exists $T>0$ such that $\bar{g}_{t} \in R^{\mathrm{inv}}(\bar{M})$ for all $t>T$.

Proof. Since $g_{\tau}$ is defined for $\tau$ in a compact interval and since $g_{\tau} \in R^{\text {inv }}(M)$ there is a constant $C>0$ so that

$$
\frac{1}{C} \int_{M}|\varphi|^{2} d v^{g_{\tau}} \leq \int_{M}\left|D^{g_{\tau}} \varphi\right|^{2} d v^{g_{\tau}},
$$




$$
\begin{gathered}
\left|\frac{1}{4} \operatorname{tr}_{g_{\tau}}\left(\dot{g}_{\tau}\right)\right|^{2} \leq C, \\
\left|g_{\tau}\left(\partial_{\tau} d v^{g_{\tau}}, d v^{g_{\tau}}\right)\right| \leq C, \\
\left|\left\langle-\frac{1}{2} \mathfrak{D}^{\dot{g}_{\tau}} \varphi+\frac{1}{4} \operatorname{grad}^{g_{\tau}}\left(\operatorname{tr}_{g_{\tau}}\left(\dot{g}_{\tau}\right)\right) \bullet_{\tau} \varphi-\frac{1}{4} \operatorname{div}^{g_{\tau}}\left(\dot{g}_{\tau}\right) \bullet_{\tau} \varphi, \varphi\right\rangle\right| \\
\quad \leq C\left(\left|\nabla^{g_{\tau}} \varphi\right|^{2}+|\varphi|^{2}\right), \\
\left|\frac{1}{4} \operatorname{scal}^{g_{\tau}}\right| \leq C .
\end{gathered}
$$

Set $\varepsilon=\frac{1}{4 C}$ and choose $T>0$ so that

$$
\frac{1}{4 C} \geq \frac{2 C^{2}+2 C+3}{4 t}+\frac{C}{t^{2}}
$$

for $t>T$.

Take $t>T$. We extend $g_{\tau}$ to $\tau \in \mathbb{R}$ by setting $g_{\tau}=g_{0}$ for $\tau<0$ and $g_{\tau}=g_{1}$ for $\tau>1$. Then $\left(\bar{M}_{\infty}, \bar{g}_{t}\right)=\left(\mathbb{R} \times M, d \tau^{2}+g_{\tau / t}\right)$. Take $\varphi \in C_{0}^{\infty}\left(\Sigma \bar{M}_{\infty}\right)$. From (1) we get

$$
\begin{aligned}
\left|D^{g_{\tau / t}} \varphi\right|^{2}+\left|\nabla_{\nu}^{\bar{g}_{t}} \varphi\right|^{2}=\mid & \left.\left(v \cdot D^{\bar{g}_{t}}-\frac{n}{2} H\right) \varphi\right|^{2} \\
& +\left\langle D^{g_{\tau / t}} \varphi, \nabla_{\nu}^{\bar{g}_{t}} \varphi\right\rangle+\left\langle\nabla_{\nu}^{\bar{g}_{t}} \varphi, D^{g_{\tau / t}} \varphi\right\rangle .
\end{aligned}
$$

When we integrate over $\bar{M}_{\infty}$ this gives

$$
\begin{aligned}
\left\|D^{g_{\tau / t}} \varphi\right\|^{2} \leq 2\left\|D^{\bar{g}_{t}} \varphi\right\|^{2}+2\left\|\frac{n}{2} H \varphi\right\|^{2} & \\
& +\int_{\bar{M}_{\infty}}\left(\left\langle D^{g_{\tau / t}} \varphi, \nabla_{\nu}^{\bar{g}_{t}} \varphi\right\rangle+\left\langle\nabla_{\nu}^{\bar{g}_{t}} \varphi, D^{g_{\tau / t}} \varphi\right\rangle\right) d v^{\bar{g}_{t}} .
\end{aligned}
$$

We are going to estimate the terms on the left-hand side of this inequality. Define the function $\theta_{\tau}=g_{\tau}\left(\partial_{\tau} d v^{g_{\tau}}, d v^{g_{\tau}}\right)$. Then $\partial_{\tau} d v^{g_{\tau / t}}=\frac{1}{t} \theta_{\tau / t} d v^{g_{\tau / t}}$. For the last term in (10) we have

$$
\begin{aligned}
\int_{\bar{M}_{\infty}} & \left(\left\langle D^{g_{\tau / t}} \varphi, \nabla_{v}^{\bar{g}_{t}} \varphi\right\rangle+\left\langle\nabla_{\nu}^{\bar{g}_{t}} \varphi, D^{g_{\tau / t}} \varphi\right\rangle\right) d v^{\bar{g}_{t}} \\
= & \int_{\mathbb{R}} \int_{\{\tau\} \times M}\left(\partial_{\tau}\left\langle D^{g_{\tau / t}} \varphi, \varphi\right\rangle-\left\langle\left[\nabla_{\nu}^{\bar{g}_{t}}, D^{\left.\left.\left.g_{\tau / t}\right] \varphi, \varphi\right\rangle\right) d v^{g_{\tau / t}} d \tau}\right.\right.\right. \\
= & \int_{\mathbb{R}}\left(\partial_{\tau} \int_{\{\tau\} \times M}\left\langle D^{g_{\tau / t}} \varphi, \varphi\right\rangle d v^{g_{\tau / t}}-\int_{\{\tau\} \times M}\left\langle D^{g_{\tau / t}} \varphi, \varphi\right\rangle \partial_{\tau} d v^{g_{\tau / t}}\right) d \tau \\
& \quad-\int_{\bar{M}_{\infty}}\left\langle\left[\nabla_{v}^{\bar{g}_{t}}, D^{g_{\tau / t}}\right] \varphi, \varphi\right\rangle d v^{\bar{g}_{t}} \\
= & -\int_{\bar{M}_{\infty}}\left(\frac{1}{t}\left\langle D^{g_{\tau / t}} \varphi, \varphi\right\rangle \theta_{\tau / t}+\left\langle\left[\nabla_{\nu}^{\bar{g}_{t}}, D^{g_{\tau / t}}\right] \varphi, \varphi\right\rangle\right) d v^{\bar{g}_{t}},
\end{aligned}
$$


so (10) becomes

$$
\begin{aligned}
\left\|D^{g_{\tau / t}} \varphi\right\|^{2} \leq 2\left\|D^{\bar{g}_{t}} \varphi\right\|^{2}+2\left\|\frac{n}{2} H \varphi\right\|^{2} & \\
& -\int_{\bar{M}_{\infty}}\left(\frac{1}{t}\left\langle D^{g_{\tau / t}} \varphi, \varphi\right\rangle \theta_{\tau / t}+\left\langle\left[\nabla_{\nu}^{\bar{g}_{t}}, D^{g_{\tau / t}}\right] \varphi, \varphi\right\rangle\right) d v^{\bar{g}_{t}} .
\end{aligned}
$$

Since $\partial_{\tau}\left(g_{\tau / t}\right)=\frac{1}{t} \dot{g}_{\tau / t}$ it follows from (6), (3) and (7) that

$$
\begin{aligned}
& \left|\int_{\bar{M}_{\infty}}\left(\frac{1}{t}\left\langle D^{g_{\tau / t}} \varphi, \varphi\right\rangle \theta_{\tau / t}+\left\langle\left[\nabla_{\nu}^{\bar{g}_{t}}, D^{g_{\tau / t}}\right] \varphi, \varphi\right\rangle\right) d v^{\bar{g}_{t}}\right| \\
& \leq \frac{C}{2 t}\left(\left\|D^{g_{\tau / t}} \varphi\right\|^{2}+\|\varphi\|^{2}\right)+\frac{C}{t}\left(\left\|\nabla^{g_{\tau / t}} \varphi\right\|^{2}+\|\varphi\|^{2}\right) .
\end{aligned}
$$

By (8) and the Lichnerowicz formula on $\left(M, g_{\tau}\right)$ we have

$$
\begin{aligned}
\left\|\nabla^{g_{\tau / t}} \varphi\right\|^{2} & =\int_{\mathbb{R}} \int_{\{\tau\} \times M}\left|\nabla^{g_{\tau / t}} \varphi\right|^{2} d v^{g_{\tau / t}} d t \\
& =\int_{\mathbb{R}} \int_{\{\tau\} \times M}\left(\left|D^{g_{\tau / t}} \varphi\right|^{2}-\frac{1}{4} \mathrm{scal}^{g_{\tau / t}}|\varphi|^{2}\right) d v^{g_{\tau / t}} d t \\
& \leq\left\|D^{g_{\tau / t}} \varphi\right\|^{2}+C\|\varphi\|^{2} .
\end{aligned}
$$

From (2) and (5) we get $\left|\frac{n}{2} H\right|^{2} \leq \frac{C}{t^{2}}$ so

$$
\left\|\frac{n}{2} H \varphi\right\|^{2} \leq \frac{C}{t^{2}}\|\varphi\|^{2} .
$$

Inserting (12), (13) and (14) into (11) we get

$$
\begin{aligned}
\left\|D^{g_{\tau / t}} \varphi\right\|^{2} \leq 2 \| & D^{\bar{g}_{t}} \varphi\left\|^{2}+2 \frac{C}{t^{2}}\right\| \varphi \|^{2} \\
& +\frac{C}{2 t}\left(\left\|D^{g_{\tau / t}} \varphi\right\|^{2}+\|\varphi\|^{2}\right) \\
& +\frac{C}{t}\left(\left\|D^{g_{\tau / t}} \varphi\right\|^{2}+C\|\varphi\|^{2}+\|\varphi\|^{2}\right)
\end{aligned}
$$

or

$$
\left\|D^{\bar{g}_{t}} \varphi\right\|^{2} \geq \frac{1}{2}\left(1-\frac{3 C}{2 t}\right)\left\|D^{g_{\tau / t}} \varphi\right\|^{2}-\left(\frac{C}{t^{2}}+\frac{C^{2}+C}{2 t}\right)\|\varphi\|^{2} .
$$

From (9) we get $1-\frac{3 C}{2 t}>0$ so (4) tells us that

$$
\begin{aligned}
\left\|D^{\bar{g}_{t}} \varphi\right\|^{2} & \geq\left(\frac{1}{2}\left(1-\frac{3 C}{2 t}\right) \frac{1}{C}-\left(\frac{C}{t^{2}}+\frac{C^{2}+C}{2 t}\right)\right)\|\varphi\|^{2} \\
& =\left(\frac{1}{2 C}-\frac{2 C^{2}+2 C+3}{4 t}-\frac{C}{t^{2}}\right)\|\varphi\|^{2} \\
& \geq \varepsilon\|\varphi\|^{2} .
\end{aligned}
$$


Since $C_{0}^{\infty}\left(\Sigma \bar{M}_{\infty}\right)$ is dense in $L^{2}\left(\Sigma \bar{M}_{\infty}\right)$ we conclude that $\bar{g}_{t} \in R^{\mathrm{inv}}(\bar{M})$.

The following corollary is immediate.

Corollary 2.4. Isotopic metrics are concordant.

2.3. Surgery. We are now going to construct a metric with invertible Dirac operator on the trace of a surgery of codimension $\geq 3$ given such a metric on the original manifold.

Let $M$ be a closed spin manifold of dimension $n$ and let $S^{n-m} \times D^{m} \rightarrow M$ be an embedding. Let $\Sigma$ be the image of $S^{n-m} \times\{0\}$. Let $W$ be the trace of the surgery on $M$ along $\Sigma$, this is constructed by attaching $D^{n-m+1} \times D^{m}$ to $M \times[0,1]$ at the image of $S^{n-m} \times D^{m} \times\{1\} \rightarrow M \times\{1\}$ and then smoothing the corner where the attaching takes place. The trace $W$ is a spin manifold with boundary $M \sqcup(\widetilde{M})^{-}$where $\widetilde{M}$ is the spin manifold obtained from $M$ by surgery along $\Sigma$.

Proposition 2.5. Assume that $W$ has been constructed from $M$ as above with $m \geq 3$. Suppose $g \in R^{\mathrm{inv}}(M)$. Then there is a metric $g^{W} \in R^{\mathrm{inv}}(W)$ such that $\left.g^{W}\right|_{M}=g$.

The proof is similar to the proof of Theorem 1.2 in [4]. We need to introduce some notation. Suppose $X$ is a submanifold of a Riemannian manifold $Y$. For $0<r$ define the distance sphere and the distance tube around $X$ as $S_{X}(r)=\{x \in Y \mid$ $\operatorname{dist}(x, X)=r\}$ and $U_{X}(r)=\{x \in Y \mid \operatorname{dist}(x, X) \leq r\}$. For $0<r_{1}<r_{2}$ define the annular region around $X$ as $A_{X}\left(r_{1}, r_{2}\right)=\left\{x \in Y \mid r_{1} \leq \operatorname{dist}(x, X) \leq r_{2}\right\}$. Let $v$ be the outward pointing unit normal of $S_{\Sigma}(r)$ and let $d A$ be the volume form of $S_{\Sigma}(r)$. In [4, Lemma 2.4] the following lemma is proved in the case where $X$ is compact, the proof also works in the formulation here.

Lemma 2.6. Let $Y$ be a Riemannian spin manifold and let $X \subset Y$ be a complete submanifold of codimension $\geq 3$ which has a uniform lower bound on the injectivity radius of its normal exponential map and for which the second fundamental form of $S_{X}(r)$ is bounded for fixed $r$.

Then there exists $0<R<1$ so that for any $0<r<\frac{1}{2} R^{11}$ and any smooth spinor field $\varphi$ defined on $A_{X}\left(r,(2 r)^{1 / 11}\right)$ satisfying

- $\int_{S_{X}(\rho)}|\varphi|^{2} d A$ is finite for all $\rho \in\left[r,(2 r)^{1 / 11}\right]$ and defines a differentiable function of $\rho$,

- $\int_{S_{X}(\rho)} \operatorname{Re}\left\langle\nabla_{\nu} \varphi, \varphi\right\rangle d A$ is finite and non-negative for all $\rho \in\left[r,(2 r)^{1 / 11}\right]$,

it holds that

$$
\|\varphi\|_{A_{X}(r, 2 r)}^{2} \leq 10 r^{5 / 2}\|\varphi\|_{A_{X}\left(r,(2 r)^{1 / 11}\right)}^{2} .
$$


Proof of Proposition 2.5. Since $g \in R^{\text {inv }}(M)$ there is an $\varepsilon^{g}>0$ so that

$$
\left\|D^{g} \varphi\right\|^{2}>\varepsilon^{g}\|\varphi\|^{2}
$$

for all $\varphi \in L^{2}(\Sigma M)$. Proposition 2.1 of [4] tells us that there is a constant $S_{0}<0$ so that for every $S_{1}>0$ there is a metric $g^{\prime}$ on $M$ which is conformal to $g$ and has the following properties:

- $g^{\prime}$ is arbitrarily close to $g$ in the $C^{1}$-topology on the space of Riemannian metrics,

- scal $^{g^{\prime}} \geq S_{0}$ on all of $M$,

- $\mathrm{scal}^{\mathrm{g}^{\prime}} \geq 2 S_{1}$ on a neighbourhood $U_{0}$ of $\Sigma$

The eigenvalues of $D^{g}$ depend continuously on the Riemannian metric with respect to the $C^{1}$-topology, see for example [3, Proposition 7.1]. We can therefore find a metric $g^{\prime}$ satisfying the above properties with $S_{1}=-8 S_{0}$ while (15) holds with the same value of $\varepsilon^{g}$. Since $g$ and $g^{\prime}$ are conformal and the dimension of the kernel of the Dirac operator is a conformal invariant we get that $g$ and $g^{\prime}$ are isotopic and bordant. So if we prove the theorem for $g^{\prime}$ we will also prove it for $g$. We replace our original $g$ with $g^{\prime}$.

Let $r>0$ be a constant so small that

- $U_{\Sigma}(2 r) \subset U_{0}$,

- $(2 r)^{1 / 11}<R$, where $R$ comes from Lemma 2.6 applied to $\Sigma \subset M$,

- $(2 r)^{1 / 11}<R$, where $R$ comes from Lemma 2.6 applied to $\mathbb{R} \times \Sigma \subset \mathbb{R} \times M$,

- $45 r^{1 / 4} \leq \varepsilon^{g}$.

Let $V$ be the trace of the surgery along $\Sigma \subset U_{\Sigma}(r)$, this trace is a manifold with boundary and codimension 2 corners. We divide the boundary of $V$ into a "horizontal" part and a "vertical" part. The horizontal part consists of $U_{\Sigma}(r) \sqcup(\widetilde{U})^{-}$where $\widetilde{U}$ is $U_{\Sigma}(r)$ after surgery along $\Sigma$. The vertical part is the cylinder $[0,1] \times \partial U_{\Sigma}(r)$. The vertical and horizontal parts meet in the two corners, which are diffeomorphic to $\partial U_{\Sigma}(r)$. From [10] we know that we can extend the metric $g$ on $M$ to a metric $g^{V}$ on $V$ without decreasing scalar curvature too much. This construction can be performed close to the surgery sphere and we get a metric on $V$ with the following properties:

- $g^{V}$ is a product metric near the horizontal part of the boundary,

- $g^{V}$ restricts to $g$ on the horizontal part $U_{\Sigma}(r)$ of the boundary,

- $g^{V}$ restricts to $d x^{2}+g$ on a neighbourhood $\cong[0,1] \times A_{\Sigma}(r-\delta, r)$ of the vertical part of the boundary,

- $\mathrm{scal}^{g^{V}} \geq S_{1}$ on $V$. 
Define $\left(W, g^{W}\right)=\left([0,1] \times\left(M-U_{\Sigma}(r)\right), d x^{2}+g\right) \cup\left(V, g^{V}\right)$ where the union is taken along the common boundary $[0,1] \times \partial U_{\Sigma}(r)$.

We first prove that $\tilde{g}=\left.g^{W}\right|_{\widetilde{M}} \in R^{\text {inv }}(\tilde{M})$. For a contradiction assume that there is a non-trivial harmonic spinor field $\varphi$ on $(\tilde{M}, \tilde{g})$. Let $\chi: M \rightarrow[0,1]$ be a cutoff function with $\chi=0$ on $U_{\Sigma}(r), \chi=1$ on $M-U_{\Sigma}(2 r)$, and $|\operatorname{grad} \chi| \leq \frac{2}{r}$. We can consider $\chi$ also a cut-off function on $\tilde{M}$ since it has support contained in $M-U=\tilde{M}-\tilde{U}$. Set $\psi=\chi \varphi$. The spinor field $\psi$ is supported in $\tilde{M}-\widetilde{U}$ where $\tilde{g}=g$ and can be considered a spinor field also for $(M, g)$.

Since scal ${ }^{\tilde{g}}=\mathrm{scal}^{g} \geq S_{1}$ on $\widetilde{U}$ and scal ${ }^{\tilde{g}}=\mathrm{scal}^{g} \geq 2 S_{1}$ on $A_{\Sigma}(r, 2 r)$, most of the norm of $\varphi$ will be concentrated away from these sets. Lemma 2.2 of [4] (a straight-forward application of the Lichnerowicz formula) tells us that

$$
\|\varphi\|_{\widetilde{U} \cup A_{\Sigma}(r, 2 r)}^{2} \leq \frac{-S_{0}}{S_{1}-S_{0}}\|\varphi\|_{\widetilde{M}}^{2}=\frac{1}{9}\|\varphi\|_{\widetilde{M}}^{2},
$$

and it follows that

$$
\|\psi\|_{M}^{2} \geq\|\psi\|_{M-U_{\Sigma}(2 r)}^{2}=\|\varphi\|_{\widetilde{M}-\left(\widetilde{U} \cup A_{\Sigma}(r, 2 r)\right)}^{2} \geq \frac{8}{9}\|\varphi\|_{\widetilde{M}}^{2} .
$$

Next we are going to show that $\varphi$ has even less norm concentrated in the annular region $A_{\Sigma}(r, 2 r)$ when compared to the larger annular region $A_{\Sigma}\left(r,(2 r)^{1 / 11}\right)$. This will follow from Lemma 2.6 and the fact that $\varphi$ is harmonic. To apply this lemma we need to show that

$$
\operatorname{Re} \int_{S_{\Sigma}(\rho)}\left\langle\nabla_{\nu}^{\tilde{g}} \varphi, \varphi\right\rangle d A \geq 0
$$

for all $\rho \in\left[r,(2 r)^{1 / 11}\right]$. Choose such a $\rho$ and set $\widehat{M}=\widetilde{U} \cup A_{\Sigma}(r, \rho)$. Then $\widehat{M}$ is a manifold with boundary $\partial \widehat{M}=S_{\Sigma}(\rho)$ and scal ${ }^{\tilde{g}} \geq S_{1}$ on $\widehat{M}$. From the Lichnerowicz formula we get

$$
\begin{aligned}
0 & =\int_{\widehat{M}}\left\langle\left(D^{\tilde{g}}\right)^{2} \varphi, \varphi\right\rangle d v^{\tilde{g}} \\
& =\int_{\widehat{M}}\left\langle\left(\nabla^{\tilde{g}}\right)^{*} \nabla^{\tilde{g}} \varphi, \varphi\right\rangle d v^{\tilde{g}}+\frac{1}{4} \int_{\widehat{M}} \operatorname{scal}^{\tilde{g}}|\varphi|^{2} d v^{\tilde{g}} \\
& \geq\left\|\nabla^{\tilde{g}} \varphi\right\|_{\widehat{M}}^{2}-\int_{\partial \widehat{M}}\left\langle\nabla_{\nu}^{\tilde{g}} \varphi, \varphi\right\rangle d A+\frac{1}{4} S_{1}\|\varphi\|_{\widehat{M}}^{2},
\end{aligned}
$$

so

$$
\operatorname{Re} \int_{\partial \widehat{M}}\left\langle\nabla_{\nu}^{\tilde{g}} \varphi, \varphi\right\rangle d A=\int_{\partial \widehat{M}}\left\langle\nabla_{\nu}^{\tilde{g}} \varphi, \varphi\right\rangle d A \geq \frac{1}{4} S_{1}\|\varphi\|_{\widehat{M}}^{2}
$$

and (17) follows since $S_{1}>0$. We now apply Lemma 2.6, which tells us that

$$
\|\varphi\|_{A_{\Sigma}(r, 2 r)}^{2} \leq 10 r^{5 / 2}\|\varphi\|_{A_{\Sigma}\left(r,(2 r)^{1 / 11}\right)}^{2} .
$$


Using this estimate we compute

$$
\begin{aligned}
\left\|D^{g} \psi\right\|_{M}^{2} & =\left\|D^{\tilde{g}}(\chi \varphi)\right\|_{\widetilde{M}}^{2}=\|\operatorname{grad} \chi \cdot \varphi\|_{\widetilde{M}}^{2} \\
& \leq \frac{4}{r^{2}}\|\varphi\|_{A_{\Sigma}(r, 2 r)}^{2} \leq 40 r^{1 / 4}\|\varphi\|_{A_{\Sigma}\left(r,(2 r)^{1 / 11}\right)}^{2} \\
& \leq 40 r^{1 / 4}\|\varphi\|_{\widetilde{M}}^{2}
\end{aligned}
$$

which together with (16) and the assumption on $r$ tells us that

$$
\left\|D^{g} \psi\right\|_{M}^{2} \leq 45 r^{1 / 4}\|\psi\|_{M}^{2} \leq \varepsilon^{g}\|\psi\|_{M}^{2},
$$

and this contradicts (15).

Let $\left(W_{\infty}, g^{W}\right)$ be $\left(W, g^{W}\right)$ with half-infinite cylindrical ends attached. Since $D^{g}$ and $D^{\tilde{g}}$ are both invertible we conclude that the essential spectrum of $D^{g^{W}}$ on $W_{\infty}$ has a gap around 0 , see for example [6, Proposition 3.24]. To prove that $g^{W} \in R^{\text {inv }}(W)$ it thus remains to show that 0 is not an eigenvalue of $D^{g^{W}}$ on $W_{\infty}$, that is to show that there are no harmonic spinors in $L^{2}\left(\Sigma W_{\infty}\right)$.

To get a contradiction assume that $\varphi \in L^{2}\left(\Sigma W_{\infty}\right)$ is a non-trivial harmonic spinor field. Then $\varphi$ is smooth and the pointwise norm decays exponentially on the cylindrical ends, see for example [6, Lemma 3.21].

Let $V_{\infty}$ be $V$ with the horizontal part of the boundary extended by half-infinite cylinders. Then $\left(W_{\infty}, g^{W}\right)=\left(\mathbb{R} \times\left(M-U_{\Sigma}(r)\right), d x^{2}+g\right) \cup\left(V_{\infty}, g^{V}\right)$ where the union is taken along the common boundary $\mathbb{R} \times \partial U_{\Sigma}(r)$. Set $\psi=(\chi \circ \pi) \varphi$ where $\chi$ is the cut-off function on $M$ defined above and $\pi: \mathbb{R} \times M \rightarrow M$ is the natural projection. The spinor field $\psi$ is supported in $W_{\infty}-V_{\infty}=\mathbb{R} \times\left(M-U_{\Sigma}(r)\right)$ where $g^{W}=d x^{2}+g$ so we can consider $\psi$ to be a spinor field on $\left(\mathbb{R} \times M, d x^{2}+g\right)$.

From [4, Lemma 2.2] applied to $V_{\infty} \cup A_{\mathbb{R} \times \Sigma}(r, 2 r) \subset W_{\infty}$ it follows that

$$
\|\psi\|_{\mathbb{R} \times M}^{2} \geq \frac{8}{9}\|\varphi\|_{W_{\infty}}^{2} .
$$

We now apply Lemma 2.6 to $\mathbb{R} \times \Sigma \subset \mathbb{R} \times M$. This can be done since $|\varphi|$ decays exponentially and since the positive scalar curvature on $V_{\infty}$ makes the computation for Equation (17) work also in this case. The conclusion is that

$$
\|\varphi\|_{A_{\mathbb{R} \times \Sigma}(r, 2 r)}^{2} \leq 10 r^{5 / 2}\|\varphi\|_{A_{\mathbb{R} \times \Sigma}\left(r,(2 r)^{1 / 11}\right)}^{2} .
$$

Using this, (18) and the assumption on $r$ we compute

$$
\begin{aligned}
\left\|D^{d x^{2}+g} \psi\right\|_{\mathbb{R} \times M}^{2} & =\left\|D^{g^{W}}(\chi \varphi)\right\|_{W_{\infty}}^{2}=\|\operatorname{grad} \chi \cdot \varphi\|_{W_{\infty}}^{2} \\
& \leq \frac{4}{r^{2}}\|\varphi\|_{A_{\mathbb{R} \times \Sigma}(r, 2 r)}^{2} \leq 40 r^{1 / 4}\|\varphi\|_{A_{\mathbb{R} \times \Sigma}\left(r,(2 r)^{1 / 11}\right)}^{2} \\
& \leq 40 r^{1 / 4}\|\varphi\|_{W_{\infty}}^{2} \leq 45 r^{1 / 4}\|\psi\|_{\mathbb{R} \times M}^{2} \\
& \leq \varepsilon^{g}\|\psi\|_{\mathbb{R} \times M}^{2}
\end{aligned}
$$


which is a contradiction since the lower bound (15) holds also for the product Dirac operator $D^{d x^{2}+g}$. We conclude that the spectrum of $D^{g^{W}}$ has a gap around 0 , and this finishes the proof of the proposition.

\section{Detecting components of $R^{\text {inv }}(M)$ using the index}

The alpha invariant of an $n$-dimensional compact spin manifold $M$ without boundary is an element $\alpha(M) \in K O_{n}(\mathbb{R})$ which only depends on the spin bordism class of $M$. The Index Theorem of Atiyah and Singer relates the alpha invariant of $M$ to an indexquantity defined using the kernel of the Dirac operator defined with respect to some metric. In particular we have the following

Proposition 3.1. Suppose $M$ is a closed spin manifold with a metric $g$ for which $D^{g}$ is invertible. Then $\alpha(M)=0$.

The first and obvious conclusion is that $R^{\text {inv }}(M)$ is empty if $\alpha(M) \neq 0$. We are going to use the alpha invariant to distinguish non-bordant metrics in $R^{\text {inv }}(M)$, for this we need some specific manifolds with non-zero alpha invariant specified in the following theorem.

Theorem 3.2. For $n=4 k+3, k \geq 1$, there are $(n+1)$-dimensional spin manifolds $Y^{i}, i \in \mathbb{Z}$, with boundary $\partial Y^{i}=S^{n}$, and metrics $g^{Y^{i}} \in R^{\mathrm{psc}}\left(Y^{i}\right), i \in \mathbb{Z}$, so that $\alpha\left(Y^{i} \cup_{S^{n}}\left(Y^{j}\right)^{-}\right)=c_{n}(i-j)$ where $c_{n} \neq 0$.

For $n=8 k$ or $n=8 k+1, k \geq 1$, there are $(n+1)$-dimensional spin manifolds $Y^{i}, i=0,1$, with boundary $\partial Y^{i}=S^{n}$, and metrics $g^{Y^{i}} \in R^{\mathrm{psc}}\left(Y^{i}\right), i=0,1$, so that $\alpha\left(Y^{1} \cup_{S^{n}}\left(Y^{0}\right)^{-}\right) \neq 0$.

Proof. In dimensions $n=4 k+3$, manifolds $\left(Y^{i}, g^{Y^{i}}\right)$ with the required properties are constructed in [13, Example 7.6, p. 328] using methods of [9].

For $n=8 k$ and $n=8 k+1$ let $Y^{0}$ be the disc $D^{n+1}$ and let $g^{Y^{0}}$ be a positive scalar curvature metric on $Y^{0}$ which is equal to the standard metric $g^{S^{n}}$ on the boundary $S^{n}$ and is product in a neighbourhood of the boundary. Let $\Sigma$ be a homotopy $(n+1)$-sphere with non-vanishing $\alpha$-invariant, see [13, Theorem 2.18, p. 93], and let $f_{0}, f_{1}: D^{n+1} \rightarrow \Sigma$ be two disjoint embedded discs. Let $W$ be $\Sigma$ with the interiors of $f_{0}\left(D^{n+1}\right)$ and $f_{1}\left(D^{n+1}\right)$ removed, then $W$ is a simply connected $h$-cobordism with boundary consisting of two components $\partial_{0} W=f_{0}\left(S^{n}\right)$ and $\partial_{1} W=f_{1}\left(S^{n}\right)$. By the $h$-Cobordism Theorem there is a diffeomorphism

$$
(F, \text { id }, f):\left([0,1] \times \partial_{0} W,\{0\} \times \partial_{0} W,\{1\} \times \partial_{0} W\right) \rightarrow\left(W, \partial_{0} W, \partial_{1} W\right) \text {. }
$$


Define $Y^{1}$ to be $\Sigma$ with the interior of $f_{1}\left(D^{n+1}\right)$ removed and identify $\partial Y^{1}$ with $S^{n}$ using $f_{1}$. Then $Y^{1}=W \cup_{\partial_{0} W} f_{0}\left(D^{n+1}\right)$. On $W$ we set $g^{Y^{1}}=\left(F^{-1}\right)^{*}\left(d x^{2}+\right.$ $\left.\left(f_{0}^{-1}\right)^{*} g^{S^{n}}\right)$ and on $f_{0}\left(D^{n+1}\right)$ we set $g^{Y^{1}}=\left(f_{0}^{-1}\right)^{*} g^{Y^{0}}$. Since $g^{Y^{0}}$ restricts to $g^{S^{n}}$ on $S^{n}$ the definitions of $g^{Y^{1}}$ fit together to a smooth metric of positive scalar curvature on $Y^{1}$. Finally

$$
\alpha\left(Y^{1} \cup_{S^{n}}\left(Y^{0}\right)^{-}\right)=\alpha\left(\left(Z-\operatorname{int} f_{1}\left(D^{n+1}\right)\right) \cup_{f_{1}\left(S^{n}\right)} f_{1}\left(D^{n+1}\right)^{-}\right)=\alpha(Z) \neq 0
$$

and we are done.

Define $h^{i} \in R^{\mathrm{psc}}\left(S^{n}\right)$ by $h^{i}=\left.g^{Y^{i}}\right|_{S^{n}}$.

Theorem 3.3. Let $M$ be a compact spin manifold of dimension $n$ and suppose $g \in$ $R^{\text {inv }}(M)$. Then

- if $n=4 k+3, k \geq 1$, there are metrics $g^{i} \in R^{\mathrm{inv}}(M), i \in \mathbb{Z}$, such that $g^{i}$ is bordant to $g$ and $g^{i}$ is not concordant to $g^{j}$ for $i \neq j$,

- if $n=8 k$ or $n=8 k+1, k \geq 1$, there is a metric $g^{1} \in R^{\text {inv }}(M)$ such that $g^{1}$ is bordant but not concordant to $g$.

Proof. We prove the theorem in the case $n=4 k+3$, the other cases are similar. Fix $i \in \mathbb{Z}$. By Proposition 2.5 there is a metric $g^{i}$ on $M \# S^{n}=M$ which is bordant to $g \sqcup h^{i}$ on $M \sqcup S^{n}$. Since the metric $h^{i}$ on $S^{n}$ is bordant to the empty manifold by the bordism $\left(Y^{i}, g^{Y^{i}}\right)$ we conclude from Corollary 2.2 that $g^{i}$ is bordant to $g$.

Denote by $\left(W^{i}, g^{W^{i}}\right)$ the bordism between $\left(M, g^{i}\right)$ and $(M, g)$ we have just constructed. The manifold $W^{i}$ is diffeomorphic to the boundary connected sum of $[0,1] \times M$ with $Y^{i}$.

Take $i, j \in \mathbb{Z}$ and suppose the metrics $g^{i}$ and $g^{j}$ are concordant. By Proposition 2.1 we can then find a metric with invertible Dirac operator on $W^{i} \cup\left(W^{j}\right)^{-}$, where the union is obtained by attaching the isometric boundary components $(M, g)$ to each other and by attaching $\left(M, g^{i}\right)$ to $\left(M, g^{j}\right)$ through a concordance of the metrics. Proposition 3.1 then tells us that $\alpha\left(W^{i} \cup\left(W^{j}\right)^{-}\right)=0$. Since $W^{i} \cup\left(W^{j}\right)^{-}$ is diffeomorphic to the connected sum of $S^{1} \times M$ and $Y^{i} \cup_{S^{n}}\left(Y^{j}\right)^{-}$we get $0=$ $\alpha\left(W^{i} \cup\left(W^{j}\right)^{-}\right)=\alpha\left(S^{1} \times M\right)+\alpha\left(Y^{i} \cup_{S^{n}}\left(Y^{j}\right)^{-}\right)=\alpha\left(Y^{i} \cup_{S^{n}}\left(Y^{j}\right)^{-}\right)=c_{n}(i-j)$ so $i=j$.

By Corollary 2.4 this result implies in dimensions $n=4 k+3$ that if $R^{\text {inv }}(M)$ is non-empty, then it has infinitely many path-components. In dimensions $n=8 k$, $8 k+1$ the result implies that if $R^{\text {inv }}(M)$ is non-empty, then it has at least two pathcomponents. We conclude that in these dimensions every closed spin manifold has a metric with non-trivial kernel of the Dirac operator, which reproves theorems by Hitchin [12, Theorem 4.5] and Bär [3, Theorem A]. 


\section{Cyclic group actions and metrics with harmonic spinors}

Let $M$ be a compact simply connected spin manifold and suppose $M \rightarrow N$ is a finite covering with covering group $G$. We do not assume that the quotient $N$ is spin or orientable. Our goal is to find metrics on $M$ with non-trivial harmonic spinors. The idea is to use the eta-invariant to show that $N$ has metrics with non-trivial harmonic spinors for generalized spin structures, and then pull such a metric back to $M$. This works under certain conditions on $G$ and $\operatorname{dim} M$, in particular we find metrics with harmonic spinors on spheres in all dimensions.

Theorem 4.1. Let $M$ be a compact simply connected spin manifold of dimension $n \geq 5$ and suppose $M \rightarrow N$ is a finite covering with covering group $\pi_{1}(N)=\mathbb{Z} / l$.

(1) If $n$ is odd assume that $N$ is orientable.

(2) If $n=2 k$ is even assume that $l=2$ and that $N$ is non-orientable with a $\mathrm{Pin}^{+}$ structure if $k$ is even or with $a$ Pin $^{-}$structure if $k$ is odd.

Then $M$ has a $\mathbb{Z} / l$-invariant metric with harmonic spinors.

The proof relies on work of Botvinnik and Gilkey [7]. Using results of [11] and [2] the argument can also be made to work with other groups and other assumptions on generalized spin structure on $N$. The proof will be given through a series of lemmas in the rest of this section.

Corollary 4.2. For $n \geq 5$ there is a metric with harmonic spinors on the sphere $S^{n}$.

Proof. We obtain a metric with harmonic spinors on $S^{n}$ by applying Theorem 4.1 to the covering $S^{n} \rightarrow P^{n}$ where $P^{n}$ is real projective space of dimension $n$. In odd dimension $P^{n}$ is orientable, in even dimension $P^{n}$ is non-orientable and has a $\mathrm{Pin}^{ \pm}$ structure as required.

4.1. Twisted spin structures and Pin structures. Following [7] we discuss twisted spin structures and Pin structures.

4.1.1. Twisted spin groups and twisted spin structures. Let $\mathbb{Z} / 2$ be the group of two elements written multiplicatively, $\mathbb{Z} / 2=\{ \pm 1\}$. Let

$$
1 \rightarrow \mathbb{Z} / 2 \rightarrow g \stackrel{\mu}{\longrightarrow} G \rightarrow 1
$$

be a central extension of a finite group $G$, this gives an action of $\mathbb{Z} / 2$ on $g$. The group $\operatorname{Spin}(n)$ is a double cover $\mathrm{SO}(n)$, identifying $\mathbb{Z} / 2$ with the kernel of the covering homomorphism gives an action of $\mathbb{Z} / 2$ on $\operatorname{Spin}(n)$. Define the twisted spin group $\mathcal{g}(g, \mu, G)=\operatorname{Spin}(n) \times \mathbb{Z} / 2 G$ where we identify $(\theta, \lambda)=(-\theta,-\lambda)$ for $\theta \in \operatorname{Spin}(n)$ and $\lambda \in G$. The twisted spin group $\mathcal{g}(g, \mu, G)$ is a double cover of $\operatorname{SO}(n) \times G$. 
Let $N$ be an $n$-dimensional oriented Riemannian manifold with oriented frame bundle $\mathrm{SO}(N)$. A $\mathcal{g}(\mathscr{g}, \mu, G)$-structure on $N$ is a principal $\mathscr{g}(\mathcal{g}, \mu, G)$-bundle $\mathcal{g}(g, \mu, G)(N)$ and an equivariant covering $g(g, \mu, G)(N) \rightarrow \mathrm{SO}(N)$ which over open sets $U$ in a suitable open cover of $N$ trivializes as $g(g, \mu, G) \times U \rightarrow \mathrm{SO}(n) \times U$. A manifold equipped with a $\mathcal{g}(\mathcal{g}, \mu, G)$-structure is called a $\mathcal{g}(\mathcal{g}, \mu, G)$-manifold. The map $\mu$ gives an extension

$$
1 \rightarrow \operatorname{Spin}(n) \rightarrow g(g, \mu, G) \stackrel{\mu}{\longrightarrow} G \rightarrow 1,
$$

through this a $\mathscr{g}(\mathcal{g}, \mu, G)$-structure $\mathcal{g}(\mathcal{g}, \mu, G)(N)$ on $N$ defines a homomorphism $\check{\mu}: \pi_{1}(N) \rightarrow G$ as the composition of the holonomy of $g(g, \mu, G)(N)$ with $\mu$. If $\check{\mu}$ is the trivial homomorphism then there is a spin structure $\operatorname{Spin}(N)$ on $N$ so that $\mathcal{g}(g, \mu, G)(N)=\operatorname{Spin}(N) \times_{\mathbb{Z} / 2} g$.

Suppose $M$ is a compact simply connected spin manifold such that $M$ is an oriented covering space of an oriented manifold $N$. Let $G=\pi_{1}(N)$ be the covering group. In [7, Theorem 1.1] a canonical $g(g, \mu, G)$-structure on $N$ with the property that the map $\check{\mu}$ is an isomorphism is constructed. The extension $(g, \mu, G)$ is given by the lift of the action of $G$ on the frame bundle $\operatorname{SO}(M)$ to the spin bundle $\operatorname{Spin}(M)$ and is split if and only if $N$ is spin. The $g(g, \mu, G)$-structure on $N$ is given by the quotient of $\operatorname{Spin}(M) \times \mathbb{Z} / 2 \&$ by $G$.

4.1.2. Spinor bundles and Dirac operators for twisted spin structures. Let $N$ be a compact oriented $n$-dimensional with a $\mathscr{g}(g, \mu, G)$-structure $\mathscr{g}(N)$. Let $h$ be a Riemannian metric on $N$. Let $\alpha$ be a unitary representation of $g$ which is odd with respect to the action of $\mathbb{Z} / 2$, that is $\alpha(-\lambda)=-\alpha(\lambda)$ for all $\lambda \in g$. We denote by $\operatorname{Rep}^{\text {odd }}(g)$ the semi-ring of odd unitary representations of $g$. Let $\Delta$ be the spinor representation of $\operatorname{Spin}(n)$, it holds that $\Delta(-\theta)=-\Delta(\theta)$ for all $\theta \in \operatorname{Spin}(n)$. Since $\Delta(\theta) \otimes \alpha(\lambda)=\Delta(-\theta) \otimes \alpha(-\lambda)$ the tensor product $\Delta \otimes \alpha$ gives a unitary representation of $g$. Let $\Sigma^{\alpha} N$ be the unitary vector bundle associated to $g(N)$ via $\Delta \otimes \alpha$, this is a bundle of twisted spinors. As with ordinary spinors there is a Clifford action by tangent vectors on $\Sigma^{\alpha} N$, and the Levi-Civita connection lifts to a connection on $\Sigma^{\alpha} N$. The Dirac operator $D^{h, \alpha}$ acting on sections of $\Sigma^{\alpha} N$ is defined as usual.

4.1.3. Pin groups and Pin structures. The Clifford algebras $\mathrm{Clif}^{ \pm}(n)$ are defined as the universal algebra with unit generated by $\mathbb{R}^{n}$ with the relations $v \cdot w+w \cdot v=$ $\pm 2(v, w)$, for $v, w \in \mathbb{R}^{n}$. The groups $\operatorname{Pin}^{ \pm}(n)$ are defined as the multiplicative subgroups of $\mathrm{Clif}^{ \pm}(n)$ generated by the unit vectors in $\mathbb{R}^{n}$. Define $\chi: \operatorname{Pin}^{ \pm}(n) \rightarrow$ $\mathbb{Z} / 2$ by $\chi\left(v_{1} \ldots v_{k}\right)=(-1)^{k}$ and $\Xi^{ \pm}: \operatorname{Pin}^{ \pm}(n) \rightarrow \mathrm{O}(n)$ by $\Xi^{ \pm}(x): v \mapsto \chi(x) x$. $v \cdot x^{-1}$. Then $\Xi^{ \pm}$are two double coverings of $\mathrm{O}(n)$ which both restrict to $\operatorname{Spin}(n) \rightarrow$ $\mathrm{SO}(n)$.

Let $N$ be an $n$-dimensional Riemannian manifold with frame bundle $\mathrm{O}(N)$. A $\operatorname{Pin}^{ \pm}$-structure on $N$ is a principal $\operatorname{Pin}^{ \pm}$-bundle $\operatorname{Pin}^{ \pm}(N)$ together with an equivariant 
covering $\operatorname{Pin}^{ \pm}(N) \rightarrow \mathrm{O}(N)$, which over open sets $U$ in a suitable open cover of $N$ trivializes as $\operatorname{Pin}^{ \pm} \times U \rightarrow \mathrm{O}(n) \times U$. A manifold equipped with a $\mathrm{Pin}^{ \pm}$-structure is called a $\mathrm{Pin}^{ \pm}$-manifold. If the set of $\mathrm{Pin}^{ \pm}$-structures on $N$ is non-empty, then the cohomology group $H^{1}(N ; \mathbb{Z} / 2)$ acts simply and transitively on this set. So on a simply connected spin manifold there are unique $\operatorname{Pin}^{ \pm}$-structures given as extensions of the unique spin structure.

4.1.4. Spinor bundles and Dirac operators for Pin structures. Let $N$ be a compact $n$-dimensional Riemannian manifold with a $\operatorname{Pin}^{ \pm}$-structure $\operatorname{Pin}^{ \pm}(N)$. Let $\Delta$ be the spinor representation of $\operatorname{Pin}^{ \pm}(n)$, and let $\Sigma N$ be the unitary vector bundle associated to $\operatorname{Pin}^{ \pm}(N)$ via $\Delta$, this is sometimes called a pinor bundle. As with ordinary spinors there is a Clifford action by tangent vectors on $\Sigma N$, and the Levi-Civita connection lifts to a connection on $\Sigma N$. The Dirac operator $D^{h}$ acting on sections of $\Sigma N$ is defined as usual.

4.1.5. Pullback to the universal covering space. Let $N$ be a compact Riemannian manifold with universal covering space $M$. Assume that $M$ is spin and that $N$ has a $\mathscr{g}$-structure $\mathscr{g}(N)$ where $\mathscr{g}=\mathscr{g}(\mathcal{g}, \mu, G)$ or $\mathscr{g}=\operatorname{Pin}^{ \pm}$. The pullback of $\mathscr{g}(N)$ to $M$ is given by an extension of the spin bundle over $M$. In case $\mathscr{g}=\mathcal{g}(\mathcal{g}, \mu, G)$ the pullback of $\Sigma^{\alpha} N$ is given by $\Sigma M \otimes \mathbb{C}^{d}$ where $d$ is the dimension of the representation $\alpha$. In case $g=\operatorname{Pin}^{ \pm}$the pullback of $\Sigma N$ is given by $\Sigma M$. In both cases the pullback of the Dirac operator on $N$ defined using some metric is given by the Dirac operator on $M$ with the pullback metric.

Let $M$ and $N$ be as in Theorem 4.1. If $n$ is odd $N$ has a $\mathcal{g}(g, \mu, G)$-structure for $G=\mathbb{Z} / l$, and we say that $(N, h)$ has harmonic spinors if $D^{h, \alpha}$ has a non-trivial kernel for some $\alpha \in \operatorname{Rep}^{\text {odd }}(\mathcal{g})$. If $n$ is even we say that $(N, h)$ has harmonic spinors if the Dirac operator $D^{h}$ associated to the $\mathrm{Pin}^{ \pm}$-structure has a non-trivial kernel. The following lemma is now obvious.

Lemma 4.3. Let $M$ and $N$ be as in Theorem 4.1. If $(N, h)$ has harmonic spinors then the pullback of $h$ to $M$ is a $\mathbb{Z} / l$-invariant metric with harmonic spinors.

4.2. Positive scalar curvature on $N$. Using known results on the Gromov-LawsonRosenberg conjecture we can prove the following lemma.

Lemma 4.4. Let $M$ and $N$ be as in Theorem 4.1. If $M$ has no $\mathbb{Z} / l$-invariant metric with harmonic spinors then $N$ has a metric of positive scalar curvature.

Proof. The Gromov-Lawson-Rosenberg conjecture for compact manifolds with finite fundamental group states the following [14, Conjecture 5.1]: A closed manifold of dimension $n \geq 5$ with finite fundamental group admits a metric with positive scalar 
curvature if and only if all index obstructions associated to Dirac operators with coefficients in flat bundles on $N$ and its covers vanish. This conjecture is known to be true in the situation at hand; for orientable manifolds with cyclic fundamental group by $[8$, Theorem 1.1] and [15, Theorem A], for non-orientable manifolds with fundamental group $\mathbb{Z} / 2$ by [14, Theorem 5.3]. So if $N$ did not have any metric with positive scalar curvature then the index and the kernel of some Dirac operator on a cover of $N$ would be non-zero. We could then take the pullback of a metric from $N$ to $M$ to produce a $\mathbb{Z} / l$-invariant metric with harmonic spinors on $M$, a contradiction.

4.3. The eta invariant. Let $M$ be a closed Riemannian manifold and let $V$ be a smooth vector bundle over $M$. Let $P$ be an operator of Dirac type acting on the space of smooth sections of $V$. For complex numbers $z$ with large real part the eta function of Atiyah, Patodi, and Singer [1] is defined as $\eta(z, P)=\operatorname{Tr}_{L^{2}}\left(P\left(P^{2}\right)^{-(z+1) / 2}\right)$. This function has a meromorphic extension to $\mathbb{C}$ for which $z=0$ is a regular value, and the eta invariant of $P$ is defined as $\eta(P)=\frac{1}{2}(\eta(0, P)+\operatorname{dim} \operatorname{ker} P)$.

For a closed Riemannian $g(g, \mu, G)$-manifold $(N, h)$ and for $\alpha \in \operatorname{Rep}^{\text {odd }}(g)$ we define $\eta(N, h, \alpha)$ as $\eta\left(D^{h, \alpha}\right)$. Let $R^{\text {odd }}(\xi)$ be the representation ring associated to $\operatorname{Rep}^{\text {odd }}(g)$ and let $R_{0}^{\text {odd }}(g)$ be the augmentation ideal consisting of virtual representations of virtual dimension 0 . The eta invariant $\eta(N, h, \alpha)$ is additive in $\alpha$ so we may extend its definition to $\alpha \in R^{\text {odd }}(g)$.

For a closed $\mathrm{Pin}^{ \pm}$-manifold $(N, h)$ we define $\eta(N, h)$ as $\eta\left(D^{h}\right)$.

Lemma 4.5. Let $M$ and $N$ be as in Theorem 4.1. Let $h^{0}, h^{1}$ be two metrics on $N$ and assume that $M$ has no $\mathbb{Z} / l$-invariant metric with harmonic spinors.

(1) If $\operatorname{dim} M$ is odd and $N$ carries a $g(g, \mu, G)$-structure then $\eta\left(N, h^{0}, \alpha\right)=$ $\eta\left(N, h^{1}, \alpha\right)$ for all $\alpha \in R_{0}^{\text {odd }}(g)$.

(2) If $\operatorname{dim} M$ is even and $N$ carries a $\operatorname{Pin}^{ \pm}{ }_{- \text {structure then }} \eta\left(N, h^{0}\right)=\eta\left(N, h^{1}\right)$.

Proof. Let $h_{\tau}, \tau \in[0,1]$, be a smooth curve of metrics on $N$ with $h_{\tau}=h^{0}$ for $\tau$ near 0 and $h_{\tau}=h^{1}$ for $\tau$ near 1 . Lemma 4.3 tells us that the Dirac operator of $h_{\tau}$ is invertible for all $\tau$. Define metrics $\bar{h}_{t}$ on $\bar{N}_{t}=[0, t] \times N$ by $\bar{h}_{t}=d \tau^{2}+h_{\tau / t}$ for $t>0$. Using the same computation as in Proposition 2.3 we conclude that $\left(\bar{N}_{t}, \bar{h}_{t}\right)$ has invertible Dirac operator for $t$ large enough when half-infinite cylinders are attached at the boundary.

First suppose that $\operatorname{dim} M$ is odd and that $N$ has a $g(g, \mu, G)$-structure. Let $\alpha \in$ $R_{0}^{\text {odd }}(g)$ be the formal difference of $\left.\alpha^{+}, \alpha^{-} \in \operatorname{Rep} \operatorname{odd}^{\text {odg }}\right)$ where $\operatorname{dim} \alpha^{+}=\operatorname{dim} \alpha^{-}$. The Atiyah-Patodi-Singer index theorem [1] tells us that

$$
\operatorname{ind}\left(D^{\bar{h}_{t}, \alpha^{ \pm}}\right)=\left(\operatorname{dim} \alpha^{ \pm}\right) \int_{\bar{N}_{t}} \hat{A}\left(g^{\bar{h}_{t}}\right)-\varepsilon\left(\eta\left(N, h^{1}, \alpha^{ \pm}\right)-\eta\left(N, h^{0}, \alpha^{ \pm}\right)\right) .
$$


Here ind $\left(D^{\bar{h}_{t}, \alpha^{ \pm}}\right)$is the index of $D^{\bar{h}_{t}, \alpha^{ \pm}}$acting on the space of sections of the positive half spinor bundle satisfying the Atiyah-Patodi-Singer boundary condition, $\hat{A}\left(\bar{h}_{t}\right)$ is the $\hat{A}$ differential form computed using the metric $\bar{h}_{t}$, and $\varepsilon= \pm 1$ is a constant depending only on the dimension. Any harmonic spinor field satisfying the AtiyahPatodi-Singer boundary conditions extends to an $L^{2}$ harmonic spinor field when half-infinite cylindrical ends are attached. Since $\bar{h}_{t}$ has invertible Dirac operator we conclude that the index is zero. We get

$$
\begin{aligned}
\eta\left(N, h^{1}, \alpha\right)-\eta\left(N, h^{0}, \alpha\right)= & \eta\left(N, h^{1}, \alpha^{+}\right)-\eta\left(N, h^{1}, \alpha^{-}\right) \\
& \quad-\eta\left(N, h^{0}, \alpha^{+}\right)+\eta\left(N, h^{1}, \alpha^{-}\right) \\
= & \varepsilon\left(\operatorname{dim} \alpha^{+}-\operatorname{dim} \alpha^{-}\right) \int_{\bar{N}_{t}} \hat{A}\left(g^{\bar{h}_{t}}\right) \\
= & 0,
\end{aligned}
$$

which proves (1).

Next suppose that $\operatorname{dim} M$ is even and that $N$ has a $\operatorname{Pin}^{ \pm}$-structure. Since $\bar{N}_{t}$ is then odd-dimensional there is no integral of a local index density in the index formula for $\left(\bar{N}_{t}, \bar{h}_{t}\right)$, and we have

$$
\operatorname{ind}\left(D^{\bar{h}_{t}}\right)=\varepsilon\left(\eta\left(N, h^{1}\right)-\eta\left(N, h^{0}\right)\right)
$$

where $\varepsilon= \pm 1$ is a constant depending only on the dimension. Again the index vanishes since $\bar{h}_{t}$ has invertible Dirac operator and we have proven (2).

4.4. Proof of Theorem 4.1. In the work [7] of Botvinnik and Gilkey the space $R^{\mathrm{psc}}(N)$ is studied for a compact manifold $N$ which is either odd-dimensional with a $g(g, \mu, G)$-structure and a finite fundamental group satisfying a certain condition or even-dimensional with fundamental group $\mathbb{Z} / 2$ and a $\operatorname{Pin}^{ \pm}$-structure. The authors construct metrics in $R^{\mathrm{psc}}(N)$ with different values of the eta invariant as follows. Assume $h \in R^{\mathrm{psc}}(N)$. First a (disconnected) manifold $\left(N^{\prime}, h^{\prime}\right)$ is found which represents zero in an appropriate bordism group and has positive scalar curvature and non-zero eta invariant. The disjoint union $N \sqcup N^{\prime}$ is then bordant to $N$ and the metric $h \sqcup h^{\prime}$ of positive scalar curvature can be extended over the bordism to give a metric $h^{1} \in R^{\mathrm{psc}}(N)$. The eta invariant is the same for psc-bordant metrics so $\eta\left(N, h^{1}\right)=\eta(N, h)+\eta\left(N, h^{\prime}\right) \neq \eta(N, h)$.

Proof of Theorem 4.1. Assume that $M$ has no $\mathbb{Z} / l$-invariant metric with harmonic spinors. From Lemma 4.4 we know that $N$ has a metric with positive scalar curvature. As discussed above, the proof of Theorem 3.1 of [7] gives us two metrics on $N$ with different $\eta$-invariant, which by Lemma 4.5 is impossible. 


\section{References}

[1] M. F. Atiyah, V. K. Patodi, and I. M. Singer, Spectral asymmetry and Riemannian geometry. I. Math. Proc. Cambridge Philos. Soc. 77 (1975), 43-69. Zbl 0297.58008 MR 0397797

[2] E. Barrera-Yanez, The eta invariant of twisted products of even-dimensional manifolds whose fundamental group is a cyclic 2 group. Differential Geom. Appl. 11 (3) (1999), 221-235. Zbl 0952.55004 MR 1726538

[3] C. Bär, Metrics with harmonic spinors. Geom. Funct. Anal. 6 (6) (1996), 899-942. Zbl 0867.53037 MR 1421872

[4] C. Bär and M. Dahl, Surgery and the spectrum of the Dirac operator. J. Reine Angew. Math. 552 (2002), 53-76, 2002. Zbl 1017.58019 MR 1940432

[5] C. Bär, P. Gauduchon, and A. Moroianu, Generalized cylinders in semi-Riemannian and Spin geometry. Math. Z. 249 (3) (2005), 545-580. Zbl 1068.53030 MR 2121740

[6] D. Bleecker and B. Booss-Bavnbek. Spectral invariants of operators of Dirac type on partitioned manifolds. In Aspects of boundary problems in analysis and geometry, Oper. Theory Adv. Appl. 151, Birkhäuser, Basel 2004, 1-130. Zbl 1067.58017 MR 2072498

[7] B. Botvinnik and P. B. Gilkey, Metrics of positive scalar curvature on spherical space forms. Canad. J. Math. 48 (1) (1996), 64-80. Zbl 0859.58026 MR 1382476

[8] B. Botvinnik and P. B. Gilkey, The Gromov-Lawson-Rosenberg conjecture: the twisted case. Houston J. Math. 23 (1) (1997), 143-160. Zbl 0884.53031 MR 1688827

[9] R. Carr, Construction of manifolds of positive scalar curvature. Trans. Amer. Math. Soc. 307 (1) (1988), 63-74. Zbl 0654.53049 MR 0936805

[10] P. Gajer, Riemannian metrics of positive scalar curvature on compact manifolds with boundary. Ann. Global Anal. Geom. 5 (3) (1987), 179-191. Zbl 0665.53040 MR 0962295

[11] P. B. Gilkey, The eta invariant of Pin manifolds with cyclic fundamental groups. Period. Math. Hungar. 36 (2-3) (1998), 139-170. Zbl 0965.58024 MR 1694601

[12] N. Hitchin, Harmonic spinors. Adv. Math. 14 (1974), 1-55. Zbl 0284.58016 MR 0358873

[13] H. B. Lawson, Jr. and M.-L. Michelsohn, Spin geometry, Princeton Math. Ser. 38, Princeton University Press, Princeton, NJ, 1989. Zbl 0688.57001 MR 1031992

[14] J. Rosenberg and S. Stolz, Manifolds of positive scalar curvature. In Algebraic topology and its applications, Math. Sci. Res. Inst. Publ. 27, Springer-Verlag, New York 1994, 241-267. Zbl 0804.57011 MR 1268192

[15] S. Stolz, Simply connected manifolds of positive scalar curvature. Ann. of Math. (2) 136 (3) (1992), 511-540. Zbl 0784.53029 MR 1189863

Received August 22, 2006

Mattias Dahl, Institutionen för Matematik, Kungl Tekniska Högskolan, 10044 Stockholm, Sweden

E-mail: dahl@math.kth.se 